\title{
A Diagnostic Approach to Bone Tumours
}

\section{Abstract}

In this review we discuss an approach to diagnosing primary bone tumours namely the cartilaginous, bone-forming, giant cell-rich, fibro-osseous and round cell neoplasms. Less common lesions including chordoma are also discussed. The value of integrating clinical, histopathological and relevant radiological features is emphasised with a view to providing the general Histopathologist with a methodical approach to reaching an accurate diagnosis. For more detailed information we recommend referring to comprehensive text book on bone tumours recently published (Czerniak, 2016) ${ }^{1}$. Key Words: Bone tumours; diagnostic approach; cartilaginous tumours; bone-forming tumours; giant cell-rich tumours; fibro-osseous tumours, round-cell tumours, notochord.

\subsection{Introduction}

Primary bone tumours are a heterogeneous group of benign and malignant neoplasms with wideranging histological appearances and genetic alterations. The clinical presentation depends on the tumour site but most are associated with pain, particularly at night. Specialist centres for rare diseases are good for patients as they benefit from a wealth of experience of a multidisciplinary team.

\subsection{Guidelines for how to approach primary bone tumours are described below}

\subsubsection{Simple histological classification}

As primary malignant bone tumours represent less than $1 \%$ of all cancers, it is best to consider in the first instance whether a tumour in bone represents either metastatic carcinoma, a germ cell tumour, a primary haematological malignancy, or the consequence of a degenerative or metabolic disorder. Once discounted, consider the morphological features and how best to classify the tumour in broad terms.

Primary bone tumour classification: Cartilage-forming, bone-forming, giant cell (osteoclast-rich), fibro-osseous, round cell and notochordal.

Many primary bone tumours comprise of a spectrum of histological phenotypes, for example, an osteosarcoma may be chondroblastic; mesenchymal chondrosarcoma exhibits a round cell 'Ewingtype' appearance with islands of chondro-osseous extracellular matrix; chondroblastoma is immature cartilage-forming with an osteoclast-rich component; and phosphaturic mesenchymal tumour exhibits a range of features and is associated with hypophosphataemia and vitamin D 
resistant rickets. Once grouped broadly on histological grounds, correlation with the clinical information and the imaging should narrow the diagnosis.

Correlation of the histological findings on a biopsy with imaging is essential: modern imaging techniques allow the histological and gross features of a tumour to be appreciated. In most circumstances the most 'worrying' areas are biopsied.

\subsubsection{Location, location, location: access to imaging is essential for musculoskeletal pathologists}

Is the tumour sited 'in the bone' (central), 'on the bone' (surface) or in the cortex?

Surface lesions: the differential diagnosis includes the various types of bone tumours described below:

Cartilage-forming: osteochondroma (connected with the underlying bone marrow), periosteal chondroma

Bone and cartilage-forming: bizarre paraosteal osteochondromatous proliferation (BPOP) (generally on the hand)

Bone-forming: a surface osteosarcoma (generally involving the tubular long bones), parosteal osteosarcoma

Fibro-osseous: fibrous dysplasia protuberans, parosteal osteosarcoma

Osteoclast-rich: aneurysmal bone cyst expanding onto the bone surface

Central bone tumours: where is the lesion sited?

Epiphyseal: giant cell tumour of bone, chondroblastoma, clear cell chondrosarcoma

Metaphyseal: osteosarcoma, conventional cartilage-forming tumours, chondromyxoid fibroma, aneurysmal bone cyst

Diaphyseal: osteoblastoma, osteofibrous dysplasia (intra-cortical), adamantinoma (intra-cortical)

These represent important examples but this is not a comprehensive list and exceptions occur 


\subsection{HISTOLOGICAL FEATURES AND TERMINOLOGY SPECIFIC TO BONE PATHOLOGY}

\section{Entrapment of host lamellar bone - a histological hallmark of malignancy}

1.3.1 Entrapment of host lamellar bone by the tumour, otherwise referred to as 'a permeative growth pattern' is a diagnostic hallmark of malignancy. This was first described by Mirra ${ }^{2}$ and reflects the speed at which a tumour is growing. The definition of 'entrapment of host lamellar bone' is that the bone exhibits Howslip's lacunae on at least three sides (Figure 1D). The absence of this finding does not exclude a malignant process and could be explained on the basis of sampling and that the tumour is growing so rapidly that the adjacent host bone is completely destroyed.

1.3.2 Pitfall: if a patient has sustained a fracture or has had previous surgery, host bone can be displaced and appear to be 'entrapped'.

1.3.3 Encasement of tumour by bone describes a benign process and is generally seen in well differentiated cartilaginous tumours. The tumour is surrounded or partly surrounded by bone and this reflects that the tumour is growing sufficiently slowly to induce bone formation from cells in the bone marrow (Figure 1C).

\subsection{CARTILAGE-FORMING TUMOURS}

1.4.1 Cartilaginous tumours (including benign and malignant types) represent the most common primary neoplasms of bone, and the most common malignant bone tumours in adults. They are classified as conventional and non-conventional. The histology of the former bares a close resemblance to non-neoplastic hyaline articular cartilage and includes enchondroma and its malignant counterpart conventional central chondrosarcoma, and osteochondroma and its malignant counterpart conventional peripheral chondrosarcoma. The non-conventional group includes chondroblastoma, chondromyxoid fibroma, dedifferentiated chondrosarcoma, mesenchymal chondrosarcoma, and clear cell chondrosarcoma.

\section{Conventional cartilaginous tumours}

\subsubsection{Enchondroma and central chondrosarcoma}

Enchondroma is the most common of all cartilaginous tumours, occurring over a wide age distribution (2nd -7 th decades). The true incidence is not known and they are often detected incidentally. They may occur in any bone but show a predilection for the small bones of the hands 
and feet: they are extremely rare at axial sites. They have a characteristic radiological appearance (Figure $1 \mathrm{~A}$ ) and correlation with these finding is often essential in reaching a definitive diagnosis. $A$ small proportion progress and transform into central conventional chondrosarcoma (Figure 1B).

1.4.3 Histologically, enchondromas demonstrate abundant hyaline matrix often encased by a rim of mature lamellar bone (Figure 1C). The chondrocytes are present in small numbers, the nuclei of which reveal a closed dense chromatin pattern and inconspicuous nucleoli. Histological and radiological features of enchondroma and chondrosarcoma grade (G) I are subtle making distinction between them nigh impossible: this is confirmed by evidence that there is considerable inter- and intra-observer variability between histopathologists making these diagnoses ${ }^{3}$. Hence we often report such tumours as 'well differentiated cartilaginous': we prefer not to use the term 'low grade chondral tumours' as grading a tumour should be restricted to malignant disease. There is good correlation between grading on needle biopsy and subsequent resection if the radiology and histology are reported by specialists ${ }^{4}$. Transition from benign (enchondroma) to malignant disease GI, GII, GIII is reflected by increasing cellularity, chondrocyte binucleation and a reduction in the hyaline matrix with accompanying myxoid change. Grade I tumours have a hyaline matrix and chondrocytes with closed pyknotic nuclei and inconspicuous nucleoli. Host bone permeation may be identified in which the residual bone trabeculae may be necrotic. Grade II tumours have a myxoid and hyaline stroma with chondrocytes showing an open chromatin pattern and conspicuous nucleoli. Grade III tumours are highly cellular with nuclear pleomorphism and a mitotic count of two or more per 10 high power fields (Figures 1D-F).

1.4.5 Chondrosarcoma of the small bones of the hands and feet is exceptionally rare ${ }^{5}$ and a definitive diagnosis in this location requires unequivocal host bone permeation with sound radiological correlation. The metastatic potential of chondrosarcoma of the small bones of the hands and feet is negligible. To reflect this, we conclude our reports with 'chondrosarcoma of the phalanges has a negligible risk of metastasis'.

\subsubsection{Clinical outcome and management}

The management of enchondromas and conventional central chondrosarcoma $\mathrm{Gl}$ in the long bones is generally curettage and the clinical outcome is excellent. Hence, for treatment purposes they can be grouped as 'well-differentiated central cartilaginous tumours'. In contrast, conventional central chondrosarcoma GII and III represent high grade disease requiring wide local excision as they have a significant risk of local recurrence if curetted, and may metastasise. Patients with chondrosarcoma GII have a $50 \%$-year survival, with a metastatic potential of $10-15 \%$. Grade III chondrosarcoma 
represents only $10-15 \%$ of all central chondrosarcomas and has an extremely poor prognosis with 5year survival rates of approximately $5 \%$, and metastatic potential of $50 \%{ }^{6}$.

\subsubsection{Isocitrate dehydrogenase type 1 (IDH1) and Isocitrate dehydrogenase type 2 (IDH2) mutations}

Mutations in Isocitrate dehydrogenase type 1 and $2(I D H 1 / 2)$ in cartilaginous tumours are highly specific for enchondromas, central conventional chondrosarcomas, periosteal chondroma/chondrosarcoma and dedifferentiated chondrosarcoma but are only seen in approximately $60 \%$ of such tumours ${ }^{7}$. These mutations are not seen in diagnostic mimics including osteochondromas, synovial chondromatosis, clear cell, peripheral and mesenchymal variants of chondrosarcoma, or osteosarcoma ${ }^{8}$.

\subsection{Osteochondroma and Conventional Peripheral Chondrosarcoma}

1.5.1 Osteochondroma (exostosis) is a benign conventional cartilaginous tumour. It is a common lesion, representing approximately $30 \%$ of all bone tumours and presents in two main forms, most commonly as a solitary lesion, but also as multiple lesions in the multiple osteochondroma (MO) syndrome ${ }^{9,10}$. Most commonly they are seen in long bones particularly at the proximal and distal ends of the femur but they can occur in almost any location. Transformation to a high-grade (peripheral) chondrosarcoma is uncommon, with high grade peripheral chondrosarcoma accounting for no more than $10-15 \%$ of all conventional chondrosarcomas ${ }^{11}$.

1.5.2 Radiologically, osteochondroma can be either a broad-based or pedunculated tumour but in all cases the stalk is in continuity with the underlying the medullary cavity hence these tumours are rarely biopsied prior to resection. X-ray only reveals the calcified and ossified component of a cartilaginous tumour and a MRI is required to reveal the uncalcified mass.

1.5.3 Osteochondroma is characterised by a mature hyaline cartilaginous cap which continues to grow until puberty, at which time the growth plate fuses, and the cap, which may reach up to $50 \mathrm{~mm}$, undergoes calcification and ossification. A narrow cartilaginous cap of a few $\mathrm{mm}$ remains and in some areas may no longer be visible (Figure 2A). Consequently, any "osteochondroma" with a cartilage cap of greater than $20 \mathrm{~mm}$ in thickness occurring in a mature skeleton is arbitrarily considered to represent a conventional peripheral chondrosarcoma (Figure 2B). However, as calcification of the cartilage cap occurs over a number of years (after puberty), such measurements must be interpreted in the context of the radiological features. 
1.5.4 The pathologist should slice the tumour to determine the thickness of the cartilage cap, and also note if the cartilage extends to the gross excision margins. Osteochondromas resected shortly after reaching skeletal maturity at puberty (up to their early 20s) may continue to reveal a hyaline cartilage cap measuring focally up to $10-15 \mathrm{~mm}$ but the presence of calcified areas is indicative of involution. Even if quite large $(50 \mathrm{~mm})$, these tumours rarely invade underlying bone. However, the presence of a myxoid and or fleshy matrix is worrying and would suggest transformation to a chondrosarcoma.

1.5.5 Histologically, osteochondromas are characterised by a mature hyaline cartilaginous cap with an underlying zone of endochondral ossification, recapitulating the organised appearance of the epiphyseal growth plate (Figure 2C). Peripheral chondrosarcoma GI can grow to a considerable size (50-100 mm) while retaining a largely hyaline matrix without invading the underlying bone. Hence, the size of the cartilage cap is important. However, the organised cellular arrangement of an osteochondroma will be lost in a peripheral chondrosarcoma and replaced by an organoid or clonal arrangement of neoplastic chondrocytes, particularly evident on the cap surface. The chondrocytes will exhibit an open chromatin pattern although this can be subtle. Management of these lesions is made at a bone tumour multidisciplinary meeting. If fully excised peripheral chondrosarcoma $\mathrm{Gl}$ is curable but if centrally placed (in the pelvis) they may be difficult to excise without morbid surgery. Incompletely excised tumours may recur and transform into high grade chondrosarcoma or a dedifferentiated chondrosarcoma (vide infra), although this is uncommon.

1.5.6 The histology of GII and GIII disease is like that of central chondrosarcoma (Figure 2D).

1.5.7 Hereditary multiple osteochondromas is an autosomal dominant disorder characterised by mutations in EXT-1 and EXT-2. Sporadic solitary osteochondromas may also harbour these mutations. ${ }^{12,13}$.

\subsection{Dedifferentiated Chondrosarcoma}

1.6.1 Dedifferentiation in a chondrosarcoma represents an aggressive complication occurring in approximately $10 \%$ of central chondrosarcomas but is extremely rare in the peripheral counterpart. The presence of a dimorphic tumour on MRI in which one component shows the features of a low grade chondral tumour (i.e. a lobular morphology with a high T2 signal) combined with features of a spindle cell sarcoma on biopsy suggests this diagnosis. Histologically, it is characterised by a conventional chondrosarcomatous component, usually GI or GIl, abutting a high-grade sarcoma with an undifferentiated appearance ${ }^{14,15}$. Heterologous elements, most commonly osteosarcomatous can be seen but rhabdomyosarcomatous and osteoclast-rich elements also occur potentially leading 
to a misdiagnosis of osteosarcoma, rhabdomyosarcoma/Triton tumour, and a giant cell tumour of bone respectively. Distinguishing between a primary osteosarcoma and a dedifferentiated chondrosarcoma usually has management implications: osteosarcoma is generally treated with neoadjuvant chemotherapy whereas this is not the case for chondrosarcoma. Radiological and demographic data can be very helpful in making the distinction however in difficult cases the identification of an IDH1 or an IDH2 mutation establishes of the diagnosis of dedifferentiated chondrosarcoma. However, these substitutions only occur in approximately $60 \%$ of such tumours ${ }^{7}$. There are now targeted therapies available against mutant $I D H 1$ and $I D H 2$ and failure to detect such mutations could potentially deny a patient being offered entry into on-going early phase clinical trials ${ }^{16}$.

\subsection{Ollier disease and Maffucci syndrome (Multiple enchondromas)}

1.7.1 Ollier disease and Maffucci syndrome represent the most common form of multiple enchondromas and are genetically related non-familial disorders. Ollier disease is characterised by multiple enchondromas, and Maffucci syndrome has the additional features of soft tissue (and more rarely visceral) spindle cell haemangiomas. Early post-zygotic somatic mutations in IDH1, and less commonly $I D H 2$, account for this mosaic disorder in virtually all cases. Hence, detection of an IDH1/2 mutation does not help grade a cartilaginous tumour occurring in these syndromic patients as it is present $a b$ initio - from enchondroma through to dedifferentiated chondrosarcoma ${ }^{7,}{ }^{17}$. Patients with these syndromes are at risk of developing chondrosarcoma in addition to many other malignancies including glioma, glioblastoma multiforme and acute myeloid leukaemia. Individuals with multiple enchondromas without IDH1/2 mutations are likely to have another form of multiple enchondromas syndrome, such as metachondromatosis (PTPN11) 18 and spondyloenchondrodysplasia (ACP5) ${ }^{19}$, and others as yet without a known genetic alteration.

\subsection{Non-conventional cartilaginous tumours}

\subsubsection{Chondroblastoma}

Chondroblastoma occurs at the epiphysis of the long bones and at apophyseal sites most commonly presenting in skeletally immature individuals. Histologically, the constituent neoplastic cell is the chondroblast (Figure 3A). Over 95\% of chondroblastomas harbour a H3F3 K36M mutation, a highly specific marker which can be detected by a highly sensitive antibody ${ }^{20,}{ }^{21}$. Chondroblastomas generally contain an osteoclast-rich component, thereby being included in the differential diagnosis of osteoclast-rich tumours ${ }^{22}$. Secondary aneurysmal bone cyst is not uncommonly associated with chondroblastoma but the presence of even a few H3.3 K36M mutant-positive cells on 
immunohistochemistry provides the diagnosis. In the absence of this characteristic mutation, chondromyxoid fibroma and phosphaturic mesenchymal tumour (PMT), the latter showing a "grungy" pattern of calcification should be considered. PMT is generally associated with low levels of blood phosphate levels and osteomalacia as a consequence of high levels of fibroblastic growth factor 23 (FGF23) which can be detected using an in situ hybridisation technology - RNAScope ${ }^{23}$.

\subsubsection{Chondromyxoid Fibroma}

Chondromyxoid fibroma is a non-conventional benign bone tumour showing cartilaginous differentiation with a predilection for the metaphysis of the tubular long bones and the flat bones of the hands and feet. It occasionally occurs within the flat bones of the pelvis where it presents as an incidental finding. Histologically, the tumour is well demarcated from the adjacent non-neoplastic bone and has a myxoid stroma with lobulation reflecting cellular and less cellular areas and true hyaline cartilage with lacuna formation is rare. The tumour cells may show nuclear pleomorphism and hyperchromasia potentially leading to an erroneous diagnosis of a conventional chondrosarcoma ${ }^{24}$. Mitoses and necrosis are exceptionally rare in chondromyxoid fibroma (Figure 3B). The differential diagnosis includes phosphaturic mesenchymal tumour: measurement of calcium and phosphate in the blood can help resolve this.

Chondromyxoid fibromas are characterised by chromothripsis on chromosome $6 q$ in more than $90 \%$ leading to upregulation of glutamate receptor metabotropic-1 (GRM1) ${ }^{25}$. As yet the identification of this complex rearrangement is not used in clinical practice for diagnostic purposes.

\subsubsection{Bizarre Paraosteal Osteochondromatous Proliferation (BPOP)}

Bizarre parosteal osteochondromatous proliferation (BPOP), also known as Nora's lesion ${ }^{26}$, is a benign, locally recurring surface tumour of the small bones of the hands and feet most commonly presenting in the $2^{\text {nd }}$ and $3^{\text {rd }}$ decades of life. Rarely it may affect the tubular long bones and less commonly the craniofacial skeleton (Figure 3C). Their often-rapid growth can give rise to clinical suspicion of malignancy, compounded by histologically complex features of a bone and cartilageforming lesion which can lead the unwary to concerns about a chondrosarcoma or an osteosarcoma. However, the site and size of a BPOP, together with the absence of atypical mitoses, host bone entrapment and a periosteal reaction generally excludes a high-grade bone sarcoma. Re-excision of a locally recurrent lesion is recommended. Malignant transformation has not been reported.

Radiologically the tumour is "stuck on" to the bone surface showing no continuity with the medullary cavity, helping to differentiate it from an osteochondroma. However imaging may not be available. 


\subsubsection{Mesenchymal chondrosarcoma}

This rare tumour occurs in bone and soft tissue. Favoured sites include the craniofacial skeleton, the ribs and vertebrae. It commonly presents in the $2^{\text {nd }}$ and $3^{\text {rd }}$ decades. The histological appearance is highly characteristic (Figure 3D). In the absence of sampling the chondro-osseous component, it is easy to misdiagnose as Ewing sarcoma. Molecular pathology tests are valuable for reaching a diagnosis, as the identification of the characteristic HEY1-NCOA2 fusion gene ${ }^{27}$ is mutually exclusive with the genetic alteration characteristic of Ewing sarcoma.

\section{BONE-FORMING TUMOURS}

\subsection{Osteoid osteoma and osteoblastoma}

1.9.1 Osteoid osteoma and osteoblastoma are benign bone-forming tumours occurring in the cortical and medullary bone respectively. They involve the metaphyseal/diaphyseal region of the tubular long bones (most commonly distal femur), the vertebral bodies, and the small bones of the hands and feet ${ }^{28}$. The pain of osteoid osteoma is characteristically relieved significantly by nonsteroidal anti-inflammatory drugs which is not the case for osteoblastoma. Both osteoid osteoma and osteoblastoma have circumscribed margins radiologically and histologically and osteoid osteoma usually has prominent associated surrounding sclerosis. Both have a central nidus which can be seen histologically and arbitrary measurements are used to distinguish them (less than 20 $\mathrm{mm}$ in the osteoid osteoma and $>20 \mathrm{~mm}$ in osteoblastoma). In both, the nidus is composed of irregular anastomosing bony trabeculae showing very prominent osteoblastic rimming with an intervening richly vascularised stroma (Figure 4) ${ }^{28}$. Osteoclasts may also be conspicuous. Osteoblastomas showing striking cellular atypia are referred to as bizarre and/or aggressive osteoblastomas but the clinical outcome does not differ to those without these atypical cells. Secondary aneurysmal bone cyst formation may occur.

\subsection{Osteosarcoma}

2.0.1 Osteosarcoma is the most common primary non-haematopoietic tumour of bone, characterised by osteoid-producing malignant cells. It rarely occurs before the age of 4 , and is most commonly seen between the ages of 10 and 14 , but $30 \%$ occur over the age of 40 . Extraskeletal osteosarcomas are classified based on anatomical location (i.e soft tissue with no skeletal continuity), and their differential diagnosis includes malignant peripheral nerve sheath tumour, and dedifferentiated liposarcoma with heterologous bone forming elements. 
2.0.2 High grade osteosarcoma of bone is treated with neoadjuvant chemotherapy: the response to treatment is one of the most important prognostic indicators of overall and disease-free survival. A good response to chemotherapy is defined as $>90 \%$ tumour necrosis post-treatment (Figure 5A, B).

2.0.3 There are various osteosarcoma subtypes classified on the basis of grade (low and high), morphology and anatomical site (central and surface). Central tumours are most common, and most are high grade: these include osteoblastic (70-80\%) chondroblastic (10-15\%), fibroblastic/pleomorphic $(10 \%)$, telangiectatic $(<4 \%)$, and rare variants (giant cell-rich, small cell, osteoblastoma-like) (Figure 5). High grade surface osteosarcoma is extremely rare and is treated the same as high grade centrally-sited tumours. Low grade osteosarcomas are also rare, representing $4 \%$ of all skeletal osteosarcomas: they arise in the medullary space (low grade central osteosarcoma, 1\%) ${ }^{29}$; and on the bone surface (parosteal osteosarcoma, 3\%). Parosteal osteosarcoma may transform to high grade disease (in $10-15 \%$ of cases), referred to as dedifferentiated parosteal osteosarcoma. Another exceptionally rare surface variant is known as periosteal osteosarcoma (vide infra).

\subsection{Tips when reporting osteosarcoma}

2.1.1 Osteoid deposition associated with highly atypical cells is the characteristic feature of highgrade osteosarcomas but the amount varies considerably. It is generally abundant in the osteoblastic type and can be minimal in the telangiectatic subtype. The morphological variants of high grade osteosarcoma do not influence the management of the disease or predict a response to treatment.

2.1.2 The large cells with low nuclear: cytoplasmic ratio in 'bizarre' osteoblastoma can be mistaken for osteosarcoma.

2.1.3 Aneurysmal bone cyst can be mistaken for telangiectatic osteosarcoma and vice versa.

2.1.4 Primary high grade fibrosarcoma or pleomorphic sarcoma in bone in which no osteoid is identified are generally managed with the same chemotherapeutic regime as an osteosarcoma. However, before making such a diagnosis it is important to exclude that the lesion does not represent a soft tissue tumour extending into bone, a dedifferentiated chondrosarcoma or metastatic disease (e.g. sarcomatoid renal cell carcinoma) from elsewhere. Imaging may help identify bone formation in cases where matrix is not noted in the biopsy, confirming a diagnosis of osteosarcoma and guiding management.

2.1.5 It can be difficult to distinguish a chondroblastic osteosarcoma from a conventional chondrosarcoma. Focussing on the degree of the chondrocyte atypia and the volume of extracellular matrix is helpful. In general, the severity of the nuclear atypia in chondroblastic osteosarcoma is 
discordant with the amount of myxoid matrix, that is there is severe atypia and a significant amount of cartilaginous matrix (Figure 5D). Imaging and demographics should be considered.

2.1.6 Immunohistochemistry is not generally helpful in reaching a diagnosis: cytokeratins, S100 and other markers can be expressed in osteosarcoma. SATB2 is not specific for osteosarcoma.

2.1.7 Small cell osteosarcoma is exceptionally rare: Ewing sarcoma, lymphoma, carcinoma, mesenchymal chondrosarcoma, and histiocytic neoplasms should be excluded before proving this diagnosis.

2.1.8 Most osteosarcomas represent primary disease, but a small percentage particularly in those presenting $>40$ years is secondary to irradiation, Pagetic disease, fibrous dysplasia, giant cell tumour of bone ${ }^{30}$, and bone infarction.

2.1.9 Approximately, $20 \%$ of patients with osteosarcoma harbour a germline genetic mutation, most commonly involving TP53 causing Li-Fraumeni syndrome, bilateral retinoblastoma syndrome, and Rothmund-Thomson syndrome ${ }^{31}$.

The somatic genetic alterations in osteosarcoma reveal that structural variants represent the predominant source of mutations and that there is a diverse landscape of driver genes: nearly $30 \%$ exhibit chromothripsis on one or more chromosomes, and a distinct copy number pattern of combined chromothripsis and amplification occurred in approximately $60 \%$ of cases $^{32}$.

\subsection{Low Grade Central Osteosarcoma}

2.2.1 Low grade central osteosarcoma most commonly occurs in the third decade of life and favours the tubular long bones, in particular the distal femur and proximal tibia. If completely excised and in the absence of an associated high grade component, the prognosis is good with only $15 \%$ of tumours metastasising. Histologically the tumour is composed of fascicles of mildly atypical spindle cells and may exhibit a fibrous dysplasia-type phenotype (Figure 5F), however as GNAS mutations are detected in $>95 \%$ of FD cases, the absence of such a mutation largely excludes this diagnosis ${ }^{33}$. In contrast, the detection of a GNAS mutation does not completely exclude a diagnosis of an osteosarcoma as $1 \%$ of fibrous dysplasia transforms into osteosarcoma. Entrapment of host lamellar bone is helpful in reaching a malignant diagnosis. Imaging is important to assess for lack of a homogeneous signal and can direct the site from where a biopsy should be taken. Following resection extensive sampling is required to exclude transformation to high grade disease. 
2.2.2 Approximately $40 \%$ of low grade central osteosarcomas harbour amplification of $M D M 2$ and this is mutually exclusive with GNAS mutations which occur in fibrous dysplasia. GNAS mutations are rarely if ever detected in low grade central osteosarcomas ${ }^{33}$.

\section{SURFACE OSTEOSARCOMA}

\subsection{Parosteal Osteosarcoma}

2.3.1 Paraosteal osteosarcoma is the most common of the surface osteosarcoma variants, occurring in adults most commonly in the third decade of life. If fully excised survival is good but reports indicate that if incompletely excised, there is a high recurrence rate, which may be many years later with transformation to high grade disease (sometimes referred to as 'dedifferentiation') and risk of metastasis. In one series, $40 \%$ of recurrences of parosteal osteosarcoma were associated with dedifferentiation ${ }^{34}$. The tumour arises directly from the external layer of the periosteum in a highly characteristic location with approximately $80 \%$ occurring on the posterior aspect of the metaphysis of the distal femur. Approximately half of parosteal osteosarcomas show cartilaginous differentiation, which can be extensive. It may be distributed throughout the tumour or take the form of a cartilaginous cap causing it to be mistaken for an osteochondroma. However, unlike an osteochondroma, parosteal osteosarcoma is 'stuck on' to the cortical bone and lacks continuity with underlying medullary bone.

2.3.2 Parosteal osteosarcoma histologically is composed of cellular fascicles of mildly to moderately atypical spindle cells interspersed with parallel bony trabeculae. The presence of a more densely cellular tumour with nuclear pleomorphism and conspicuous mitotic activity is characteristic of transformation to high grade disease. However, distinguishing low from high grade disease is subjective and not always easy. High grade disease is associated with a more aggressive course and a poorer prognosis. Genetically parosteal osteosarcoma, irrespective of grade, shows amplification of MDM2 and CDK4 in approximately $85 \%$ of cases ${ }^{35}$; to date there is no objective biomarker defining transformation to high grade disease.

\subsection{Periosteal Osteosarcoma}

2.4.1 Periosteal osteosarcoma is an exceptionally rare variant of osteosarcoma: it is an intermediate grade surface tumour most commonly occurring in the second and third decades of life. It shows a predominant chondroblastic morphology, and osteoid is usually focal. There is no characteristic genetic alteration reported. Periosteal osteosarcoma has a better prognosis as compared to conventional osteosarcoma with approximately $80 \%$ of patients surviving long term ${ }^{36}$ and has 
therefore come to be classified as an intermediate grade tumour. This rare tumour is managed with neo-adjuvant chemotherapy followed by surgical resection.

\subsection{OSTEOCLAST CELL-RICH TUMOURS}

Assessment of serum calcium and phosphate levels should be undertaken in all osteoclast-rich lesions to exclude hyperparathyroidism and other metabolic diseases where a definitive diagnosis based on molecular findings has not been proven.

The differential diagnosis of giant cell tumours includes aneurysmal bone cyst, chondroblastoma, giant cell granuloma of the jaw and small bones of the hand and feet, brown tumour of hyperparathytroidism, fibrous dysplasia, Pagetic bone disease and osteoclast-rich lesions of the jaw seen in germline disorders including Cherubism, Noonan syndrome, and Neurofibromatosis $37,38$.

Multiple osteoclast-rich tumours require further investigation to exclude hyperparathyroidism and germline conditions. Multiple giant cell tumours with H3F3A G34W mutations has been recently reported to occur as part of a mosaic disorder in conjunction with paragangliomas and other tumours. Multiple giant cell tumour-type/non-ossifying fibroma-type lesions can also occur in the presence of the linear naevus sebaceous syndrome, associated with HRAS/KRAS mutations.

\subsection{Giant cell tumour of bone}

2.6.1 Giant cell tumour of bone is a locally aggressive and rarely metastasising tumour, occurring in the epiphysis of the long bones. It is exceptional to see this tumour in the immature skeleton. Most present in the second to fourth decades. Favoured sites are the distal femur and proximal tibia followed by the distal radius and the sacrum.

2.6.2 The most common treatment is curettage and cementation however if tumour recurs or involves the joint, a wide resection with a prosthetic implant may be required. Denosumab, a RANKL inhibitor, is now employed for the treatment of giant cell tumours in surgically inaccessible sites such as the pelvis and sacrum, and base of skull. This inhibitor blocks recruitment of osteoclasts resulting in maturation of the neoplastic cells and bone formation. The histological changes associated with Denosumab many lead to the erroneous diagnosis of osteosarcoma ${ }^{39}$.

2.6.3 Histologically, giant cell tumours of bone are composed of large osteoclasts containing up to 100 nuclei with interspersed mononuclear cells, representing the neoplastic component (Figure 6A). 
These cells express high levels of receptor activator nuclear factor K-B (RANKL), which accounts for the recruitment of the osteoclast-like giant cells as a non-neoplastic reactive population. Mitotic figures may be seen in large numbers although the presence of atypical forms raises concerns of a sarcoma. More than $95 \%$ of giant cell tumours of bone harbour a p.G34 point mutation in the H3F3A gene. This mutation is only detected in the mononuclear cell component, confirming these as the neoplastic element of giant cell tumour of bone (Figure $6 \mathrm{~B}$ ). The vast majority of mutations are represented by p.G34W but p.G34L occurs in small numbers. The former can be detected by immunohistochemistry, simplifying the diagnosis of these tumours ${ }^{40}$. The antibody is highly sensitive and specific for this diagnosis ${ }^{41}$. However as approximately $2 \%$ of osteosarcomas also harbour a H3.3 G34 mutation it is not possible to rely purely on the presence of this alteration for making a diagnosis of giant cell tumour of bone: morphological, radiological and clinical correlation remain essential ${ }^{41}$.

2.6.4 The main challenge to making a diagnosis of giant cell tumour of bone is to distinguish it from an osteoclast-rich osteosarcoma. For this, histological assessment remains the gold standard: osteoclast-rich osteosarcoma and malignant giant cell tumour of bone show significant atypia in the mononuclear cell component, often with atypical mitoses, not seen in giant cell tumours of bone. Rarely, a dedifferentiated chondrosarcoma exhibits an osteoclast-rich component: detection of an IDH1/2 mutation allows the diagnosis to be made but the absence of such a mutation is not helpful.

\subsection{Primary aneurysmal bone cyst (ABC)}

2.7.1 $\mathrm{ABC}$ is an intramedullary osteolytic tumour presenting in any bone over a wide age range but usually in patients under 30 . Genetically it is characterised by a USP6 fusion gene in $70 \%$ of cases. As there are a wide range of fusion partners, FISH is the best clinical test available at present for detection of the USP6 rearrangement. An identical molecular signature also occurs in nodular fasciitis ${ }^{42}$.

2.7.2 Histologically, the tumour is composed of solid and cystic areas in varying proportions: there are blood-filled spaces surrounded by with fibrous septae often containing osteoid seams, sometimes exhibiting a blue hue, referred to as 'blue bone' (Figure 6C). Secondary aneurysmal bone cyst is a frequent reactive morphological appearance associated with any primary bone tumour, although most commonly seen with benign lesions. This component does not harbour the USP6 alteration seen in the primary $A B C$. The presence of secondary bone cyst change can account for rapid and worrying expansion of a benign tumour. 


\subsection{Non-ossifying fibroma}

2.8.1 Non-ossifying fibroma (metaphyseal fibrous defect) is a fibrohistiocytic lesion occurring in the metaphyseal region of tubular long bones, typically in skeletally immature individuals with a characteristic imaging appearance Image. Favoured sites include the distal femur and the proximal and distal ends of the tibia. Histologically, non-ossifying fibroma is composed of bland spindleshaped cells and macrophages with a storiform architecture (Figure 6D). They often undergo spontaneous involution, raising the possibility that they represent a reactive rather than neoplastic process. See section on multifocal osteoclast-rich lesions ${ }^{37,38}$.

\subsection{Giant cell-rich lesions of the craniofacial skeleton}

2.9.1 A wide range of giant cell rich lesions occur in the craniofacial skeleton, some of which are associated with specific diseases with germline alterations, namely neurofibromatosis/Jaffe Campannacci syndrome (NF1), Cherubism (SH3BP2), and Noonan/Leopard syndrome (most commonly PTPN11, but also KRAS, BRAF, HRAS, and others) ${ }^{43}$. Central and peripheral reparative giant cell granulomas are distinguished radiologically by continuity with the bone, and pure soft tissue involvement, respectively. If a central giant cell granuloma recurs or behaves aggressively, consideration should be given to whether the patient may harbour one of the aforementioned germline disorders. The lesions occurring on the background of one of these germline disorders exhibit similar morphologies to a non-ossifying fibroma. However, the histological spectrum extends to those found in giant cell tumour of bone. Giant cell tumour of bone (defined as harbouring a H3.3 G34W mutation) has not been reported in the jaw ${ }^{44}$.

\section{FIBRO-OSSEOUS TUMOURS}

\subsection{Fibrous Dysplasia}

3.0.1 Fibrous dysplasia is a central benign neoplastic fibro-osseous tumour presenting in monostotic $(75 \%)$ or polyostotic $(25 \%)$ forms. Any bone can be affected and the imaging is characterised by a 'ground glass' appearance on x-ray. The lesion should not 'break through' the cortical bone although this can occur in 'thin' bones such as the skull, ribs and small bones of the hand. Presenting symptoms include pain, deformity, fracture and cranial nerve defects, depending on the bone involved.

3.0.2 Fibrous dysplasia is associated with hypophosphatemia in $10 \%$ of cases hence calcium and phosphate should be measured in all affected patients ${ }^{45}$. We like to include this recommendation in 
our pathology report. The hypophosphatemia is caused by fibroblastic growth factor 23 being secreted by tumour cells and exerting a systemic effect on the kidneys. This can result in bowing of the legs and skeletal deformities in the immature/growing skeleton and requires treatment. Referral to a metabolic bone specialist is recommended.

3.0.3 Histologically, fibrous dysplasia is characterised by bland monotonous spindle cells in which variable amounts of immature woven bone (appreciable on polarisation) is seen, arranged in irregular angulated and curvilinear trabeculae - so-called "Chinese characters." The bony trabeculae lack osteoblastic rimming (in contrast to osteofibrous dysplasia - vide infra) ${ }^{46}$. Mitotic figures are not common, although more so in children, and should consequently arouse suspicion of malignancy.

3.0.4 The main differential diagnosis of fibrous dysplasia is a low grade central osteosarcoma, and if protruding out of the medullary cavity, a parosteal osteosarcoma. GNAS mutations and MDM2 amplification are mutually exclusive, helping to distinguish these lesions ${ }^{33}$. Osteofibrous dysplasia should always be considered in the differential diagnosis in the tibia and fibula, and immunohistochemistry for broad-spectrum cytokeratins always performed. The presence of cytokeratin expression and GNAS mutations are mutually exclusive. Fibrous dysplasia may also be associated with intra-muscular myxoma, Mazabraud syndrome, which also harbours the GNAS mutation as part of the mosaic disorder.

3.1 Osteofibrous dysplasia is a benign cortical-based tumour occurring essentially only in the tibia and fibula. It may be associated with a $C M E T$ germline mutation, when it presents bilaterally in young patients ${ }^{47}$. These mutations have not been detected in the sporadic variant of the disease. Histologically, it is very similar to fibrous dysplasia but is distinguished by osteoblastic rimming of the bony trabeculae and the presence of cytokeratin-positive cells, a feature which is mutually exclusive with the presence of GNAS mutations. Osteofibrous dysplasia is considered to be the precursor of adamantinoma, although this transition is a rare event.

\section{NOTOCHORDAL TUMOURS}

\subsection{Chordoma}

3.2.1Chordoma is a malignant tumour showing notochordal differentiation and is considered to originate from persistent midline embryonic notochord elements. Chordoma is a sporadic disease in the vast majority of cases and may exceptionally be inherited as an autosomal dominant trait, brought about by tandem duplication of the brachyury gene, otherwise known as $T$. Chordoma occurs in the axial skeleton from the spheno-occipital region/base of skull to the sacro-coccygeum: it occurs very occasionally in extra-axial bone, and soft tissue sites ${ }^{48}$. Chordoma presents at all ages 
from birth but most commonly in the 5th and 6th decades. In the paediatric age group, they occur most commonly in the spheno-occital region and the cervical spinal column. The median survival is 7 years. Metastases present late, usually several years after initial presentation, with spread to other bones and unusual sites such as the subcutaneous tissue. The mainstay of management is surgery in association with particles or photons, allowing higher doses of radiation ${ }^{49}$. EGFR inhibitors have been identified as a potential new therapeutic option ${ }^{50}$.

3.2.2 Most chordomas exhibit a typical histology (Figure 7A), and given their clear cell morphology, the differential diagnosis includes metastatic carcinoma and chondrosarcoma. The diagnostic challenge is compounded by the fact that chordoma expresses cytokeratin ${ }^{51}$. However, brachyury expression, the diagnostic hallmark of chordoma except in the dedifferentiated variant, is rarely seen in carcinoma (Figures 7B and7C) ${ }^{49}$. Chondroid differentiation may be noted but these cells also express brachyury and this morphology does not appear to influence the clinical outcome or treatment.

3.2.3 Benign notochordal cell tumour (BNCT) arises in the same anatomical regions as chordoma ${ }^{52}$. Radiologists can generally make the diagnosis on imaging and therefore they are rarely biopsied. There is no consensus on how to monitor patients in whom these lesions are identified. Histologically they can be mistaken for adipose tissue but express brachyury and cytokeratins (Figure 7D). Occasionally these are found adjacent to chordoma providing supportive evidence that they represent a precursor tumour. BNCT has a notochordal phenotype but does not show permeation into the host bone or soft tissue involvement. Ecchordosis physaliphoroa is a benign notochordal cell tumour occurring in the spheno-occipitalial region.

\section{ROUND CELL TUMOURS}

\subsection{EWING SARCOMA AND EWING SARCOMA FAMILY OF TUMOURS (ESFT)}

A diagnosis of a 'round cell tumour' should rarely be given today as most tumours with this morphology can be classified on the basis of a genetic event and or immunohistochemistry.

Most ESFT harbour an EWSR1 rearrangement, however EWSR1 alterations are found in many other tumour types including clear cell sarcoma, angiomatoid fibrous histiocytoma, myoepithelioma, extraskeletal myxoid chondrosarcoma and desmoplastic small round cell tumour. As such, morphological evaluation is paramount.

3.3.1 Ewing sarcoma is a malignant "small round cell" tumour showing neuroectodermal differentiation. Previously sub-classified as peripheral neuroectodermal tumours (PNET), Askin tumours and others these are now collectively referred to as the Ewing sarcoma family of tumours 
(ESFT). They occur at all sites, both in bone and soft tissue, although $80 \%$ of those in bone present in children whereas the soft tissue tumours mainly occur in adults. They are typically sited in the metaphyseal/diaphyseal region of the tubular long bones of the extremities. Clinically the tumour may mimic osteomyelitis presenting with pain, fever, weight loss and leucocytosis. The prognosis of Ewing sarcoma has dramatically improved since the advent of modern chemo-therapeutic regimes in the 1980s but little thereafter. The 5-year survival rates for localised tumours is approximately 70 $80 \%$ but only $15-30 \%$ for tumours which are metastatic at presentation ${ }^{53}$.

3.3.2 Histologically, Ewing sarcomas exhibit a monotonous "small round cell" appearance with membranous immunoreactivity for CD99 but they occasionally show a more pleomorphic morphology (atypical Ewing sarcoma), with less prominent CD99 expression (Figure 8A, B). CD99 has poor specificity and positivity should be combined with identification of a recurrent genetic alteration for diagnostic confirmation of Ewing sarcoma. 90\% harbour an EWS-FLI1 fusion gene (Figure $8 \mathrm{C}$ ). Other fusion partners have been described, including ERG and ETV1. In addition, there are tumours referred to as Ewing-like sarcomas representing part of the ESFT lacking the characteristic molecular signature. These tumours show a predominantly round cell appearance although with a more varied morphology: they are characterised by translocations in CIC-DUX4, and BCOR-CCNB3 (Figure 8D). The latter occurs in bone. Although it is a contentious issue as to whether these tumours belong to the Ewing family of sarcomas, they are currently treated with the same neoadjuvant chemotherapy regimens ${ }^{54}$. There are new potential emerging diagnostic markers detectible by immunohistochemistry such as NKX2-2, a downstream target of EWSR1-FLI1. This marker is sensitive but not specific for the diagnosis of Ewing sarcoma, being expressed in other tumours including mesenchymal chondrosarcoma, CIC-DUX4 and BCOR-CCNB3 rearranged round cell sarcomas, meaning it may have limited diagnostic utility ${ }^{55}$.

\section{ACKNOWLEDGMENT}

Adrienne Flanagan is a National Institute for Health Research senior investigator and is supported by the National Institute for Health Research, UCLH Biomedical Research Centre, and the CRUK UCL Experimental Cancer Centre. Daniel Lindsay is a Health Education England Research Fellow.

Figure 1: Enchondromas and conventional central chondrosarcoma. 
A. Enchondroma (MRI): a well-defined expansile lobular chondral tumour within the proximal aspect of the proximal phalanx of the left fifth toe showing a characteristic punctate pattern of calcification without exciting a periosteal reaction, or extending into soft tissues.

B. Chondrosarcoma GII (MRI): a patient with Ollier's disease showing multiple chondral tumours. The largest tumour is present at the superior aspect of the left iliac crest and left supra-acetabular region. The tumour has a heterogeneous appearance with osseous destruction and an accompanying periosteal reaction.

C. Enchondroma (H\&E X40): a cartilaginous tumour with a hyaline matrix containing evenly distributed chondrocytes with closed pyknotic nuclei (closed chromatin pattern) that may be mistaken for mitotic figures. The mature cartilaginous tumour is encased by a rim of lamellar bone: no host bone permeation is noted $(*)$.

D. Conventional central chondrosarcoma, GI (H\&E X40): a cartilaginous tumour with a hyaline matrix showing slight disorganisation of the chondrocytes, but the nuclei exhibit a closed chromatin pattern. Extensive host bone permeation is noted with formation of Howslip's lacunae appreciated, confirming malignancy $(*)$.

E. Conventional central chondrosarcoma, GII (H\&E x100): a hypercellular cartilaginous tumour with myxoid stromal change and spindled tumour cells with an open chromatin pattern and prominent nucleoli.

F. Conventional central chondrosarcoma, GIII (H\&E x200): highly atypical cells with an open chromatin pattern and hyperchromasia. Mitotic activity is easily appreciated. Two or more figures/10 high power fields classifies the tumour as grade III ${ }^{56}$.

\section{Figure 2: Osteochondroma and conventional peripheral chondrosarcoma.}

A. Osteochondroma: an outer fibrous peri-chondrial layer with an underlying hyaline cartilaginous cap, showing variable calcification. The uncalcified portion of the cartilaginous cap measures $<20 \mathrm{~mm}$, a reassuring feature supporting a benign lesion.

B. Conventional peripheral chondrosarcoma: the uncalcified portion of the cartilaginous cap is $>20 \mathrm{~mm}$ with an irregular nodular configuration: this is indicative of malignant transformation in skeletally mature individuals.

C. Osteochondroma, (H\&E x20): a mature hyaline cartilaginous cap featuring organised chondrocytes with a closed chromatin pattern and underlying endochondral ossification. No permeation into the underlying medullary bone is seen. The uncalcified portion of the cartilaginous cap is marked with a double-headed arrow. Only this portion of the cartilaginous cap is considered when assessing for malignant transformation. 
D. Conventional peripheral chondrosarcoma, GII (H\&E x40): note the hyaline stroma and disorganised chondrocytes. The uncalcified portion of the cartilaginous cap in this case measures $35 \mathrm{~mm}$.

\section{Figure 3: Non-conventional cartilaginous tumours.}

A. Chondroblastoma (H\&E x100). Oval neoplastic chondroblasts with eosinophilic cytoplasm are distributed throughout. Note the focal calcification, which if extensive, imparts a socalled characteristic "chicken wire" appearance. Inset: Immunohistochemistry for H3F3 K36M showing nuclear positivity, confirming the diagnosis.

B. Chondromyxoid fibroma (H\&E x100). The tumour cells have a spindled to stellate morphology with amphopihilic cytoplasm and a closed chromatin pattern set in a myxoid cartilaginous matrix. Peripheral cellularity imparts a pseudo-lobular appearance. No mitotic figures or necrosis is appreciable, helping to differentiate this tumour from a chondrosarcoma, GII. This can be confirmed by radiological correlation.

C. BPOP (H\&E X20). A surface-based cellular fibro-osseous cartilaginous tumour showing the osseous component here with a strong haemotoxyphilic 'blue' staining pattern. Note there is no cytological atypia. The lesions are small and often scraped off thus the histology is disrupted and imaging may not be available.

D. Mesenchymal chondrosarcoma (H\&E x40). A bi-phasic tumour represented by welldifferentiated cartilage and primitive monotonous malignant "small round blue cells". A prominent haemangiopericytomatous vascular pattern is also noted.

\section{Figure 4: Osteoid osteoma}

Microscopy (H\&E x40). The figure demonstrates a central nidus of an osteoid osteoma. Plump osteoblasts and conspicuous osteoclasts line the bone trabeculae. Between the trabeculae there is conspicuous vascularity. The nidus is surrounded but more mature bone (arrow) present at the periphery. These features are also seen in osteoblastoma. When interpreted in the context of the radiological features, this tumour should not be misdiagnosed as osteosarcoma.

\section{Figure 5: Conventional Osteosarcoma.}


A. A high grade osteoblastic osteosarcoma post-chemotherapy-treated distal femoral resection. A metaphyseal-diaphyseal-sited tumour with extension to the epiphysis. Cortical breach with periosteal elevation (Codman's triangle) and soft tissue extension are appreciated.

B. The percentage of response to pre-operative chemotherapy requires histological assessment of a complete cross section of the specimen (bone slab).

C. High grade osteoblastic osteosarcoma (H\&E x100). Tumour cells with a high-grade morphology with marked nuclear pleomorphism and atypical mitoses. Osteoid deposition is extensive.

D. High grade chondroblastic osteosarcoma (H\&E x100). Malignant tumour with chondroblastic features showing a myxo-hyaline cartilaginous matrix with pleomorphic tumour cells. The degree of nuclear atypia combined with the hyaline quality of the matrix favours a diagnosis of chondroblastic osteosarcoma. Osteoid deposition varies but is sparse in this case.

E. High grade fibroblastic osteosarcoma (H\&E x40). Osteoid production with pleomorphic tumour cells set in a densely collagenised stroma. Host bone entrapment is noted with formation of Howslip's lacunae $(* *)$.

F. Low grade central osteosarcoma (H\&E x40). Mildly atypical spindle cells arranged in a fascicular architecture and irregular bony trabeculae showing entrapment of adjacent host bone.

\section{Figure 6 Osteoclast Cell Rich Tumours.}

A. Giant cell tumour of bone (H\&E x100): The neoplastic mononuclear cells are oval with moderate amounts of eosinophilic cytoplasm and occasionally prominent nucleoli. Osteoclast-like giant cells are evenly distributed throughout. There is no cytological atypia within the mononuclear cells or atypical mitoses, helping to exclude an osteoclast-rich osteosarcoma. Scattered typical mitoses may be seen.

B. G34W Immunohistochemistry (H\&E x100): Nuclear immunoreactivity within the mononuclear cells confirming the presence of a H3.3 G34W mutation. Note the lack of reactivity in the osteoclast-like giant cells serve as an internal negative control.

C. Aneurysmal bone cyst (H\&E x40): Cystic tumour in which the neoplastic component is represented by inconspicuous monotonous spindle cells. Not the osseous metasplasia with a characteristic blue hue so-called "blue bone" $(*)$. Inset: FISH for USP6 using a break apart probe showing a split signal, confirming the presence of a rearrangement. 
D. Non-ossifying fibroma (H\&E x100): Metaphyseal lesion composed of monomorphic spindle cells with a fibro-histiocytic morphology with a storiform architecture associated with numerous foamy histiocytes and scattered osteoclast-like giant cells: the inset shows histological detail. No significant cytological atypia is seen.

\section{Figure 7: Notochordal Cell Tumours.}

A. Chordoma (H\&E x100): The tumour consists of cords of cells with well-defined cytoplasmic boundaries, foamy or bubbly cytoplasm and prominent nucleoli. The bubbly quality gives rise to the characteristic term "physaliphorous cell". There are intervening fibrous septae and myxoid changes in the background stroma.

B. Brachyury Expression (IHC x100): Strong nuclear positivity helping to distinguish the tumour from a metastatic clear cell carcinoma and chondrosarcoma.

C. Dedifferentiated chordoma (H\&E X40): Chordoma with an adjacent undifferentiated spindle cell tumour. Identification of the differentiated component in combination with the radiological features and characteristic axial location all support the diagnosis.

D. Benign Notochordal Cell Tumour (H\&E x40): Tumour composed of clear cells with bland nuclear features and a notochordal phenotype. There is no cytological atypia, myxoid change or necrosis, allowing a confident distinction to be made in most instances. This tumour was seen incidentally following resection of a sacral osteosarcoma.

\section{Figure 8 The Ewing Sarcoma Family of Tumours (ESFT)}

A. Ewing sarcoma (H\&E x100): Tumour with a monotonous "small round cell" morphology composed of cells with scanty cytoplasm. Homer-Wright rosettes may be present, indicative of neuroectodermal differentiation.

B. Immunohistochemistry for CD99 (H\&E x100): Strong membranous staining characteristic of Ewing sarcoma. This marker has low specificity and must be interpreted in the context of the radiological and morphological findings.

C. Break-apart probe for the EWSR1 gene from the case shown in figure 8A: red and green signals split apart indicating a positive result, confirming the diagnosis of Ewing sarcoma. 
D. CIC-DUX4 rearranged sarcoma (H\&E x100): A malignant tumour with a "small round cell" morphology. Note that the cells show a more variable degree of pleomorphism and are less monotonous that in conventional Ewing sarcoma.

\section{References}

1. Czerniak B. Dorfman and Czerniak's Bone Tumors [electronic resource]. 2nd ed. ed. Philadelphia, PA: Elsevier Health Sciences; 2016.

2. Mirra JM, Gold R, Downs J, Eckardt JJ. A new histologic approach to the differentiation of enchondroma and chondrosarcoma of the bones. A clinicopathologic analysis of 51 cases. Clin Orthop Relat Res. 1985:214-237.

3. Eefting D, Schrage YM, Geirnaerdt MJ, et al. Assessment of interobserver variability and histologic parameters to improve reliability in classification and grading of central cartilaginous tumors. Am J Surg Pathol. 2009;33:50-57.

4. Roitman PD, Farfalli GL, Ayerza MA, Múscolo DL, Milano FE, Aponte-Tinao LA. Is Needle Biopsy Clinically Useful in Preoperative Grading of Central Chondrosarcoma of the Pelvis and Long Bones? Clin Orthop Relat Res. 2017;475:808-814.

5. Dahlin DC, Salvador AH. Chondrosarcomas of bones of the hands and feet--a study of 30 cases. Cancer. 1974;34:755-760.

6. Nota SP, Braun Y, Schwab JH, van Dijk CN, Bramer JA. The Identification of Prognostic Factors and Survival Statistics of Conventional Central Chondrosarcoma. Sarcoma. 2015;2015:623746.

7. Amary MF, Bacsi $K$, Maggiani $F$, et al. IDH1 and IDH2 mutations are frequent events in central chondrosarcoma and central and periosteal chondromas but not in other mesenchymal tumours. J Pathol. 2011;224:334-343.

8. Damato $\mathrm{S}$, Alorjani $\mathrm{M}$, Bonar $\mathrm{F}$, et al. IDH1 mutations are not found in cartilaginous tumours other than central and periosteal chondrosarcomas and enchondromas. Histopathology. 2012;60:363-365.

9. Kitsoulis P, Galani V, Stefanaki K, et al. Osteochondromas: review of the clinical, radiological and pathological features. In Vivo. 2008;22:633-646.

10. Bovée JV. Multiple osteochondromas. Orphanet J Rare Dis. 2008;3:3.

11. Garrison RC, Unni KK, McLeod RA, Pritchard DJ, Dahlin DC. Chondrosarcoma arising in osteochondroma. Cancer. 1982;49:1890-1897.

12. Jäger $M$, Westhoff $B$, Portier $S$, et al. Clinical outcome and genotype in patients with hereditary multiple exostoses. J Orthop Res. 2007;25:1541-1551.

13. Jennes I, de Jong D, Mees K, Hogendoorn PC, Szuhai K, Wuyts W. Breakpoint characterization of large deletions in EXT1 or EXT2 in 10 multiple osteochondromas families. BMC Med Genet. 2011;12:85.

14. Frassica FJ, Unni KK, Beabout JW, Sim FH. Dedifferentiated chondrosarcoma. A report of the clinicopathological features and treatment of seventy-eight cases. J Bone Joint Surg Am. 1986;68:1197-1205.

15. Johnson S, Têtu B, Ayala AG, Chawla SP. Chondrosarcoma with additional mesenchymal component (dedifferentiated chondrosarcoma). I. A clinicopathologic study of 26 cases. Cancer. 1986;58:278-286.

16. Fujii $\mathrm{T}$, Khawaja MR, DiNardo $\mathrm{CD}$, Atkins JT, Janku F. Targeting isocitrate dehydrogenase (IDH) in cancer. Discov Med. 2016;21:373-380.

17. Amary MF, Damato $S$, Halai $D$, et al. Ollier disease and Maffucci syndrome are caused by somatic mosaic mutations of IDH1 and IDH2. Nat Genet. 2011;43:1262-1265. 
18. Yang W, Wang J, Moore DC, et al. Ptpn11 deletion in a novel progenitor causes metachondromatosis by inducing hedgehog signalling. Nature. 2013;499:491-495.

19. Bhargava R, Leonard NJ, Chan AK, Spranger J. Autosomal dominant inheritance of spondyloenchondrodysplasia. Am J Med Genet A. 2005;135:282-288.

20. Behjati S, Tarpey PS, Presneau N, et al. Distinct H3F3A and H3F3B driver mutations define chondroblastoma and giant cell tumor of bone. Nat Genet. 2013;45:1479-1482.

21. Amary MF, Berisha F, Mozela R, et al. The H3F3 K36M mutant antibody is a sensitive and specific marker for the diagnosis of chondroblastoma. Histopathology. 2016;69:121-127.

22. de Silva MV, Reid R. Chondroblastoma: varied histologic appearance, potential diagnostic pitfalls, and clinicopathologic features associated with local recurrence. Ann Diagn Pathol. 2003;7:205-213.

23. Carter JM, Caron BL, Dogan A, Folpe AL. A novel chromogenic in situ hybridization assay for FGF23 mRNA in phosphaturic mesenchymal tumors. Am J Surg Pathol. 2015;39:75-83.

24. Rahimi A, Beabout JW, Ivins JC, Dahlin DC. Chondromyxoid fibroma: a clinicopathologic study of 76 cases. Cancer. 1972;30:726-736.

25. Nord KH, Lilljebjörn H, Vezzi F, et al. GRM1 is upregulated through gene fusion and promoter swapping in chondromyxoid fibroma. Nat Genet. 2014;46:474-477.

26. Nora FE, Dahlin DC, Beabout JW. Bizarre parosteal osteochondromatous proliferations of the hands and feet. Am J Surg Pathol. 1983;7:245-250.

27. Wang L, Motoi $T$, Khanin R, et al. Identification of a novel, recurrent HEY1-NCOA2 fusion in mesenchymal chondrosarcoma based on a genome-wide screen of exon-level expression data. Genes Chromosomes Cancer. 2012;51:127-139.

28. Atesok KI, Alman BA, Schemitsch EH, Peyser A, Mankin H. Osteoid osteoma and osteoblastoma. J Am Acad Orthop Surg. 2011;19:678-689.

29. Kurt AM, Unni KK, McLeod RA, Pritchard DJ. Low-grade intraosseous osteosarcoma. Cancer. 1990;65:1418-1428.

30. Amary F, Berisha F, Ye H, et al. H3F3A (Histone 3.3) G34W Immunohistochemistry: A Reliable Marker Defining Benign and Malignant Giant Cell Tumor of Bone. Am J Surg Pathol. 2017.

31. Zhang J, Nichols KE, Downing JR. Germline Mutations in Predisposition Genes in Pediatric Cancer. N Engl J Med. 2016;374:1391.

32. Behjati $S$, Tarpey $P$, Haase $K$, et al. Recurrent mutation of IGF signalling genes and distinct patterns of genomic rearrangement in osteosarcoma. Nat. Commun. 2017;Accepted for publication.

33. Salinas-Souza C, De Andrea C, Bihl M, et al. GNAS mutations are not detected in parosteal and low-grade central osteosarcomas. Mod Pathol. 2015;28:1336-1342.

34. Sheth DS, Yasko AW, Raymond AK, et al. Conventional and dedifferentiated parosteal osteosarcoma. Diagnosis, treatment, and outcome. Cancer. 1996;78:2136-2145.

35. Dujardin F, Binh MB, Bouvier C, et al. MDM2 and CDK4 immunohistochemistry is a valuable tool in the differential diagnosis of low-grade osteosarcomas and other primary fibroosseous lesions of the bone. Mod Pathol. 2011;24:624-637.

36. Rose PS, Dickey ID, Wenger DE, Unni KK, Sim FH. Periosteal osteosarcoma: long-term outcome and risk of late recurrence. Clin Orthop Relat Res. 2006;453:314-317.

37. Colby RS, Saul RA. Is Jaffe-Campanacci syndrome just a manifestation of neurofibromatosis type 1? Am J Med Genet A. 2003;123A:60-63.

38. Mankin HJ, Trahan CA, Fondren G, Mankin CJ. Non-ossifying fibroma, fibrous cortical defect and Jaffe-Campanacci syndrome: a biologic and clinical review. Chir Organi Mov. 2009;93:17.

39. Roitman PD, Jauk F, Farfalli GL, Albergo JI, Aponte-Tinao LA. Denosumab-Treated Giant Cell Tumor of Bone Its Histologic Spectrum and Potential Diagnostic Pitfalls. Hum Pathol. 2017.

40. Presneau N, Baumhoer D, Behjati $S$, et al. Diagnostic value of H3F3A mutations in giant cell tumour of bone compared to osteoclast-rich mimics. J Pathol Clin Res. 2015;1:113-123. 
41. Amary F, Berisha F, Ye H, et al. H3F3A (Histone 3.3) G34W immunohistochemistry: a reliable marker defining benign and malignant giant cell tumour of bone. Am. J. Surg. Pathol. 2017; Accepted for publication.

42. Erickson-Johnson MR, Chou MM, Evers BR, et al. Nodular fasciitis: a novel model of transient neoplasia induced by MYH9-USP6 gene fusion. Lab Invest. 2011;91:1427-1433.

43. Idowu BD, Thomas G, Frow R, Diss TC, Flanagan AM. Mutations in SH3BP2, the cherubism gene, were not detected in central or peripheral giant cell tumours of the jaw. Br J Oral Maxillofac Surg. 2008;46:229-230.

44. Flanagan AM, Speight PM. Giant cell lesions of the craniofacial bones. Head Neck Pathol. 2014;8:445-453.

45. Collins MT, Singer FR, Eugster E. McCune-Albright syndrome and the extraskeletal manifestations of fibrous dysplasia. Orphanet J Rare Dis. 2012;7 Suppl 1:S4.

46. Reed RJ. Fibrous dysplasia of bone. A review of 25 cases. Arch Pathol. 1963;75:480-495.

47. Gray MJ, Kannu P, Sharma S, et al. Mutations Preventing Regulated Exon Skipping in MET Cause Osteofibrous Dysplasia. Am J Hum Genet. 2015;97:837-847.

48. McMaster ML, Goldstein AM, Bromley CM, Ishibe N, Parry DM. Chordoma: incidence and survival patterns in the United States, 1973-1995. Cancer Causes Control. 2001;12:1-11.

49. Stacchiotti S, Sommer J, Group CGC. Building a global consensus approach to chordoma: a position paper from the medical and patient community. Lancet Oncol. 2015;16:e71-83.

50. Scheipl S, Barnard M, Cottone L, et al. EGFR inhibitors identified as a potential treatment for chordoma in a focused compound screen. J Pathol. 2016;239:320-334.

51. Tirabosco R, Mangham DC, Rosenberg AE, et al. Brachyury expression in extra-axial skeletal and soft tissue chordomas: a marker that distinguishes chordoma from mixed tumor/myoepithelioma/parachordoma in soft tissue. Am J Surg Pathol. 2008;32:572-580.

52. Bjornsson J, Wold LE, Ebersold MJ, Laws ER. Chordoma of the mobile spine. A clinicopathologic analysis of 40 patients. Cancer. 1993;71:735-740.

53. Ladenstein R, Pötschger $U$, Le Deley $M C$, et al. Primary disseminated multifocal Ewing sarcoma: results of the Euro-EWING 99 trial. J Clin Oncol. 2010;28:3284-3291.

54. Antonescu C. Round cell sarcomas beyond Ewing: emerging entities. Histopathology. 2014;64:26-37.

55. Hung YP, Fletcher CD, Hornick JL. Evaluation of NKX2-2 expression in round cell sarcomas and other tumors with EWSR1 rearrangement: imperfect specificity for Ewing sarcoma. Mod Pathol. 2016;29:370-380.

56. Evans HL, Ayala AG, Romsdahl MM. Prognostic factors in chondrosarcoma of bone: a clinicopathologic analysis with emphasis on histologic grading. Cancer. 1977;40:818-831. 